\title{
The Green's functions of a vertically inhomogeneous soil with a random dynamic shear modulus
}

\author{
M. Schevenels *, G. Lombaert, G. Degrande, \\ D. Degrauwe, B. Schoors \\ K.U.Leuven, Department of Civil Engineering, \\ Kasteelpark Arenberg 40, B-3001 Leuven, Belgium
}

\begin{abstract}
This paper deals with the study of the Green's functions of a layered soil with random characteristics. The dynamic shear modulus of the soil is modelled as a nonGaussian random process that varies in the vertical direction and is characterized by a marginal probability density function and a correlation function. The stochastic finite element method is applied to a hybrid thin layer - direct stiffness formulation in order to obtain the stochastic system equations, which are solved by means of a Monte Carlo simulation. The influence of the variations of the dynamic shear modulus on the Green's functions is illustrated for different excitation frequencies and receiver positions.
\end{abstract}

Key words: Green's functions, Stochastic finite element method, Non-Gaussian random process, Monte Carlo simulation.

\section{Introduction}

Vibrations induced by road, rail, and subway traffic are a common source of discomfort to people. Numerical models have been developed for the prediction of traffic induced vibrations in the free field or in the built environment $[1,2,3]$. These models consist of a finite element formulation for the vehicles and the buildings and a boundary element formulation that accounts for the wave propagation in the soil. The latter is based on the Green's functions of a

* Corresponding author: mattias.schevenels@bwk.kuleuven.be, tel: + 32163216 82, fax: + 3216321988 . 
horizontally layered halfspace. The prediction models have been validated for both road traffic and rail traffic [4]. For road traffic, the dominant frequency is about $10 \mathrm{~Hz}$ and a good correspondence of the predicted and the measured response is observed. For rail traffic, however, the dominant frequency is about $50 \mathrm{~Hz}$ and the validation reveals a discrepancy between the predicted and the measured response in the higher frequency range. Given the crucial role of the Green's functions in the prediction model, the dynamic soil characteristics governing these functions are a possible source of the discrepancy.

The most common techniques for the in situ measurement of the dynamic soil characteristics include the spectral analysis of surface waves (SASW) test, the seismic cone penetration test (SCPT), the crosshole test, and the downhole test. These techniques allow one to estimate the variation of the dynamic shear modulus and the material damping ratio with depth. The SASW test and the SCPT test are complementary: the SASW test has a better accuracy at shallow depth, while the SCPT test achieves a better accuracy at larger depth. Both techniques are based on the measurement of local averages of the dynamic soil characteristics, resulting in a limited resolution in the sense that small scale variations of the dynamic soil characteristics are not revealed. The cone tip resistance measured in a cone penetration test (CPT) is the result of averaging over a smaller spatial scale. Therefore, it is possible to derive additional information on the small scale variations of the dynamic soil characteristics if a relation with the cone tip resistance is assumed.

In the present paper, the influence of the (small scale) variations of the dynamic shear modulus on the Green's functions of a soil excited at the surface is examined. While the importance of the variation of the material damping ratio is also recognized, the influence is not accounted for in the present paper as the in situ measurement techniques required to assess this variation are still in an early stage of development. As it is practically impossible to formulate an accurate deterministic description of the soil profile that accounts for the small scale variations of the dynamic shear modulus, a probabilistic approach is followed: the Green's functions are calculated for a layered soil where the dynamic shear modulus is modelled as a random process that varies in the vertical direction. The fact that the formation of a soil layer is governed by large scale phenomena such as erosion, transport, and weathering processes is put forward as an argument to neglect smaller scale variations in the horizontal direction [5].

In the field of structural mechanics, a well-established numerical method for problems where the characteristics are modelled as random processes is provided by the stochastic finite element method (SFEM) developed by Ghanem and Spanos [6]. This method relies on the discretization of random processes by means of the Karhunen-Loeve (KL) decomposition: a process is expressed as a linear combination of deterministic functions with random coefficients. While 
the KL decomposition is usable in a direct way for Gaussian processes, recently developed techniques $[7,8]$ allow for a similar decomposition of non-Gaussian processes, extending the applicability of the SFEM. A parallel decomposition is performed at the level of the finite element equations to obtain the stochastic system equations. These are solved by means of a Monte Carlo simulation, a Neumann expansion, or a projection of the response on the polynomial chaos.

Yeh and Rahman [9] apply the SFEM for the site response analysis of a layered soil modelled with finite elements. The dynamic shear modulus is modelled as a Gaussian process that varies in the vertical direction. As the response is dominated by vertically propagating shear waves, a one-dimensional model suffices. The stochastic system equations are solved by means of a Monte Carlo simulation, a Neumann expansion, and a projection of the response on the polynomial chaos.

Ghiocel and Ghanem [10] present a procedure for the probabilistic analysis of the seismic soil-structure interaction problem. The earthquake ground acceleration, the low strain shear modulus of the soil, and the dependence of the shear modulus on the shear strain are modelled as non-Gaussian random processes, where the shear modulus is assumed to vary only in the vertical direction. The Young's modulus and the material damping of the structure are modelled as random variables characterized by a mean value and a standard deviation. The SFEM is used to assemble the stochastic system equations. A hybrid method is applied for the solution of these equations where the projection of the response on the polynomial chaos is estimated by means of a stratified sampling technique.

Liao and Li [11] use a two-dimensional finite element model for the calculation of the seismic response of a layer on rigid bedrock where the Young's modulus is modelled as a random process. Absorbing boundary conditions are employed in order to fulfill Sommerfeld's radiation conditions. The stochastic system equations are solved by means of a projection of the response on the polynomial chaos.

Nour et al. [12] use a similar model for the calculation of the seismic response of a layer on rigid bedrock. The soil characteristics are modelled as bivariate random processes. The lognormal probability density function (PDF) is used for the dynamic shear modulus while the Beta PDF is used for both the material damping ratio and the Poisson's ratio. The stochastic response is calculated by means of a Monte Carlo simulation where realizations of the non-Gaussian soil characteristics are obtained as transformations of underlying Gaussian processes.

In this paper, the methodology of the SFEM is applied to a hybrid thin layer direct stiffness formulation [13] in order to obtain the stochastic system equa- 
tions for a soil subjected to a vertical harmonic load. The thin layer method is used to model the upper part of the soil where the dynamic shear modulus is modelled as a univariate non-Gaussian process. This method is based on the discretization of the displacement field in the vertical direction and the analytical solution of the wave equation in the horizontal direction, fulfilling Sommerfeld's radiation conditions without the need for absorbing boundaries. The direct stiffness method is used to model the underlying halfspace with deterministic characteristics. This method is based on the analytical solution of the wave equation for a homogeneous layer and a homogeneous halfspace. The resulting stochastic system equations are solved in the frequency-wavenumber domain by means of a Monte Carlo simulation, yielding the stochastic Green's functions of the soil.

In order to illustrate the methodology and to demonstrate how the excitation frequency and the source-receiver distance affect the mean value and the variance of the free field response, the stochastic Green's functions are calculated for a site in Sint-Katelijne-Waver (Belgium). The focus is on the influence of the variations of the dynamic shear modulus. The Poisson's ratio, the material damping ratio and the density of the soil are modelled as deterministic and spatially invariant variables, resulting in a stochastic model that only partially reflects the variability of the soil. While this approach reveals the influence of the dynamic shear modulus, it does not allow for the validation of the numerical results by means of supplementary in situ measurements.

\section{Stochastic soil characterization}

A site in Sint-Katelijne-Waver (Belgium) is considered where the soil consists of relatively homogeneous stiff Boom clay. Table 1 summarizes the soil profile as derived from SASW and laboratory tests up to a depth $L=14 \mathrm{~m}$ [14]. For each layer, the thickness $d$, the dynamic shear modulus $G$, the Poisson's ratio $\nu$, the density $\rho$, and the hysteretic material damping ratio $\beta$ are given, as well as the corresponding shear and dilatational wave velocities $C_{s}$ and $C_{p}$.

Table 1

\begin{tabular}{c|ccccccc} 
Layer & $\begin{array}{c}d \\
{[\mathrm{~m}]}\end{array}$ & $\begin{array}{c}G \\
{[\mathrm{MPa}]}\end{array}$ & $\begin{array}{c}\nu \\
{[-]}\end{array}$ & $\begin{array}{c}\rho \\
{\left[\mathrm{kg} / \mathrm{m}^{3}\right]}\end{array}$ & $\begin{array}{c}\beta \\
{[-]}\end{array}$ & $\begin{array}{c}C_{s} \\
{[\mathrm{~m} / \mathrm{s}]}\end{array}$ & $\begin{array}{c}C_{p} \\
{[\mathrm{~m} / \mathrm{s}]}\end{array}$ \\
\hline 1 & 1.8 & 47.3 & 0.33 & 1988 & 0.01 & 170 & 338 \\
2 & 4.3 & 230.8 & 0.47 & 1988 & 0.01 & 340 & 1429 \\
3 & $\infty$ & 439.1 & 0.47 & 1988 & 0.01 & 470 & 1975
\end{tabular}

Measured soil profile at the site in Sint-Katelijne-Waver. 


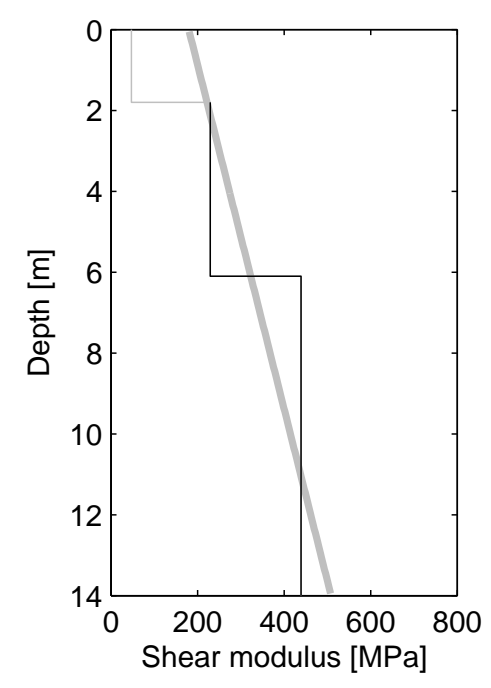

Fig. 1. Dynamic shear modulus derived from SASW tests.

The SASW test results suggest a soil profile consisting of two homogeneous layers on top of a homogeneous halfspace as shown in figure 1. This profile is different in nature from the cone tip resistance shown in figure 2 that has been recorded for $N_{\mathrm{cpt}}=30 \mathrm{CPTs}$ at the same site [14]. Assuming the existence of a relation between the dynamic shear modulus and the cone tip resistance, the CPT results reveal a dynamic shear modulus that exhibits small scale variations in the vertical direction.

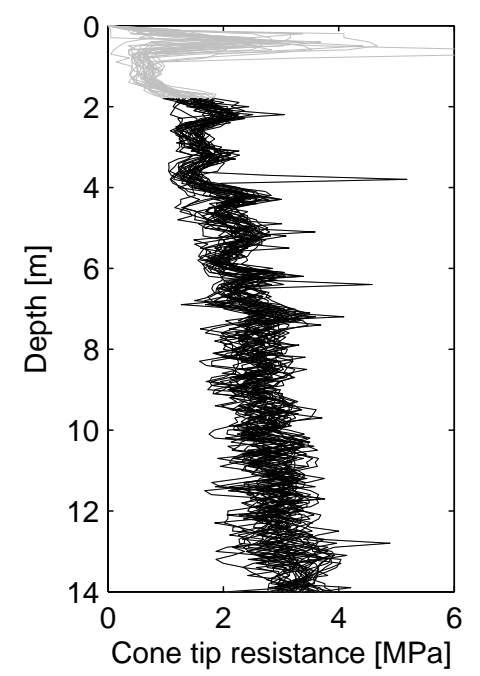

Fig. 2. Cone tip resistance for 30 CPTs.

The results from the SASW tests and the CPTs are combined to derive a stochastic representation for the dynamic shear modulus $G(z, \theta)$ in the domain $D=\{z: 0 \leq z \leq L\}$. Herein, $\theta$ is the coordinate in the random dimension and $z$ is the vertical coordinate in a cylindrical frame of reference $(r, \phi, z)$ where the origin is located at the soil's surface and the $z$-axis is pointing downwards. Following Fenton [15], the dynamic shear modulus $G(z, \theta)$ is expressed as a 
linear memoryless transformation of a second order stationary process $Y(z, \theta)$ with zero mean and unit variance:

$$
G(z, \theta)=m_{G}(z)+\sigma_{G}(z) Y(z, \theta)
$$

where $m_{G}(z)$ and $\sigma_{G}(z)$ denote the mean value and the standard deviation of the dynamic shear modulus $G(z, \theta)$.

The mean value $m_{G}(z)$ of the dynamic shear modulus is derived from the SASW tests. For the site in Sint-Katelijne-Waver, the thicknesses of the layers revealed by the SASW tests are not directly related to the real stratification of the soil. They are rather related to the basis functions used to discretize the soil profile in the inversion of the SASW measurement data. Furthermore, the top layer consists of disturbed soil. The properties of this layer vary in the horizontal direction and it is beyond the scope of the present study to account for these lateral variations. Therefore, a linear profile as shown in figure 1 is assumed:

$$
m_{G}(z)=a z+b
$$

The coefficients $a=23.5 \mathrm{MPa} / \mathrm{m}$ and $b=179.8 \mathrm{MPa}$ are determined by means of a least squares approximation of the SASW profile in the domain $D$ where the properties of the disturbed top layer are assumed to be identical to the properties of the second layer. The corresponding wave velocities of the mean system increase from $C_{s}=301 \mathrm{~m} / \mathrm{s}$ and $C_{p}=1264 \mathrm{~m} / \mathrm{s}$ at the surface $z=0 \mathrm{~m}$ to $C_{s}=506 \mathrm{~m} / \mathrm{s}$ and $C_{p}=2126 \mathrm{~m} / \mathrm{s}$ at a depth $z=14 \mathrm{~m}$.

The second order properties of the dynamic shear modulus $G(z, \theta)$ are derived from the CPTs, disregarding the measurement results in the disturbed top layer. This requires a relation between the dynamic shear modulus $G$ and the cone tip resistance $q_{c}$. Whereas a wide range of empirical relations is available in the literature [16], the following simplified relation is used in this paper:

$$
G=\alpha q_{c}
$$

The coefficient $\alpha=145.9$ is obtained by means of a least squares approximation of the mean dynamic shear modulus $m_{G}(z)$ derived from the SASW tests versus the cone tip resistance $q_{c}(z)$ resulting from the CPTs.

The CPT profiles are considered as realizations $q_{c}\left(z, \theta_{i}^{\prime}\right)$ of the random process $q_{c}(z, \theta)$ and transformed into realizations $G\left(z, \theta_{i}^{\prime}\right)$ of the dynamic shear modulus according to equation (3). The index $i$ refers to the $i$-th CPT. The coefficient of variation $\sigma_{G}(z) / m_{G}(z)$ of the dynamic shear modulus $G(z, \theta)$ is assumed not to vary as a function of the depth. Assuming ergodicity and second order stationarity of the process $G(z, \theta) / m_{G}(z)$, the standard deviation 
$\sigma_{G}(z)$ is estimated as:

$$
\sigma_{G}(z)=m_{G}(z) \sqrt{\frac{1}{N_{\mathrm{cpt}}} \sum_{i=1}^{N_{\mathrm{cpt}}} \frac{1}{L} \int_{0}^{L}\left[\frac{G\left(z^{\prime}, \theta_{i}^{\prime}\right)}{m_{G}\left(z^{\prime}\right)}-1\right]^{2} d z^{\prime}}=0.16 m_{G}(z)
$$

The realizations of the dynamic shear modulus $G\left(z, \theta_{i}^{\prime}\right)$ are transformed into realizations of the zero mean unit variance process $Y\left(z, \theta_{i}^{\prime}\right)$ according to equation (1). The correlation function of the process $Y(z, \theta)$ is assumed to decay exponentially:

$$
C_{Y}(\Delta z)=\exp \left(-\Delta z / l_{c}\right)
$$

Following Fenton [15], the correlation length $l_{c}=0.42 \mathrm{~m}$ is estimated as the area under the sample correlation function $\hat{C}_{Y}(\Delta z)$ of the realizations $Y\left(z, \theta_{i}\right)$. Both the sample correlation function $\hat{C}_{Y}(\Delta z)$ and the exponential correlation function $C_{Y}(\Delta z)$ are shown in figure 3 .

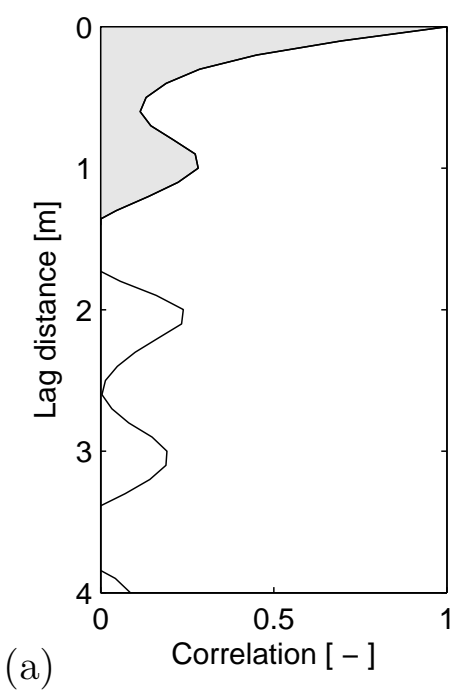

Fig. 3. (a) Sample correlation function $\hat{C}_{Y}(\Delta z)$ and (b) exponential correlation function $C_{Y}(\Delta z)$ of the random process $Y(z, \theta)$.

The marginal $\operatorname{PDF} p_{Y}(y)$ of the zero mean unit variance process $Y(z, \theta)$ is chosen so that the dynamic shear modulus $G(z, \theta)=m_{G}(z)+\sigma_{G}(z) Y(z, \theta)$ assumes a Gamma marginal PDF.

The random process $Y(z, \theta)$ is not completely defined as it is only characterized by a marginal PDF and a correlation function. This kind of incomplete stochastic characterization is common in the literature as the inference of the complete family of joint PDFs of a random process is practically impossible.

The remaining dynamic soil properties, such as the material damping ratio, may also vary as a function of the depth. These characteristics are modelled 
as deterministic and spatially invariant variables, however, as the focus in the present paper is on the influence of the variations of the dynamic shear modulus. The Poisson's ratio is equal to $\nu=0.47$, the hysteretic damping ratio is $\beta=0.01$ and the density is $\rho=1988 \mathrm{~kg} / \mathrm{m}^{2}$. The dynamic shear modulus of the underlying halfspace $(z \geq L)$ is equal to $m_{G}(z=L)=509 \mathrm{MPa}$.

\section{Numerical model}

This section focuses on the numerical model for the calculation of the stochastic Green's functions $\hat{u}_{i j}^{G}\left(\mathbf{x}^{S}, \mathbf{x}^{R}, \omega, \theta\right)$ of a layered soil with a random dynamic shear modulus $G(z, \theta)$. The Green's functions represent, in the frequency domain, the displacement at the receiver position $\mathbf{x}^{R}$ in the direction $\mathbf{e}_{j}$ due to a concentrated harmonic load $\rho \hat{b}_{k}(\mathbf{x}, \omega)=\delta\left(\mathbf{x}-\mathbf{x}^{S}\right) \delta_{i k}$ at the source position $\mathbf{x}^{S}$ in the direction $\mathbf{e}_{i}$. The tensor $\delta_{i k}$ denotes the Kronecker delta and the function $\delta(\diamond)$ denotes the Dirac delta function. In the present case, the focus is on the displacements due to a vertical harmonic load applied at the origin of the coordinate system, located at the soil's surface. Hence, the problem exhibits axial symmetry and the notation of the Green's functions is reduced to $\hat{u}_{z j}^{G}(r, z, \omega, \theta)$ where $(r, z)$ are the coordinates of the receiver.

First, the hybrid thin layer - direct stiffness formulation [13] is briefly reviewed. This formulation allows one to obtain the Green's functions of a soil where the dynamic shear modulus is represented by a deterministic function of the depth: the dynamic shear modulus is discretized so that a layered soil profile is obtained that consists of homogeneous thin layers on top of a homogeneous halfspace. Next, it is shown how the random process $G(z, \theta)$ is decomposed into a linear combination of deterministic functions with random coefficients. Finally, the deterministic soil model and the decomposition of the random process are combined in order to obtain a stochastic system of algebraic equations that leads to the Green's functions of a layered soil with a randomly varying dynamic shear modulus.

\subsection{Hybrid thin layer - direct stiffness formulation}

The thin layer method (TLM) is an efficient technique for the analysis of wave propagation in media with material characteristics that vary only in the vertical direction $z$ [13]. Following a discretization scheme analogous to the finite element method (FEM), the displacement field is discretized in the vertical direction while the wave equation is solved analytically in the horizontal direction. 
The direct stiffness method (DSM) is an alternative to the TLM where the wave equation is solved analytically for each individual layer or for a homogeneous halfspace $[13,17]$. Whereas the DSM is very efficient for a soil consisting of a small number of thick homogeneous layers, the TLM is more efficient in the present case where the dynamic shear modulus varies continuously as a function of the depth and the number of layers is large. A hybrid method is therefore applied where the TLM is used to model the soil in the domain $D$ with a varying dynamic shear modulus $G(z, \theta)$ and the DSM is used to model the underlying halfspace.

In order to reduce the partial differential equations to a system of algebraic equations, the system equations are formulated in the frequency-wavenumber domain:

$$
\tilde{\mathbf{K}} \tilde{\mathbf{U}}=\tilde{\mathbf{F}}
$$

where a tilde denotes the dependency on the frequency $\omega$ and the radial wavenumber $k_{r}$. The dynamic soil stiffness matrix $\tilde{\mathbf{K}}$ results from the assembly of the stiffness matrices of the layers and the homogeneous halfspace. The vectors $\tilde{\mathbf{F}}$ and $\tilde{\mathbf{U}}$ denote the external traction applied to each layer interface and the displacement of each interface, respectively.

The system equations are solved for a vertical harmonic load applied at the origin of the coordinate system in order to obtain the Green's functions $\tilde{u}_{z j}^{G}\left(k_{r}, z, \omega, \theta\right)$ in the frequency-wavenumber domain. An inverse Hankel transformation leads to the Green's functions $\hat{u}_{z j}^{G}(r, z, \omega, \theta)$ in the frequency-spatial domain. The transformation is performed by means of a fast Hankel transform algorithm, using a logarithmic sampling of the wavenumber $k_{r}$ and the radial coordinate $r$. The improved algorithm developed by Van Veldhuizen [18] is used to compute the vertical displacement at the receivers located below the free surface, while the original algorithm developed by Talman [19] is used otherwise.

\subsection{Decomposition of the random process}

In order to formulate the stochastic system equations, the zero mean nonGaussian process $Y(z, \theta)$ is discretized by means of the KL decomposition [6] of order $M^{\prime}$ :

$$
Y(z, \theta) \approx \sum_{j=1}^{M^{\prime}} \sqrt{\lambda_{j}^{\prime}} f_{j}^{\prime}(z) \xi_{j}^{\prime}(\theta)
$$

Herein, $\lambda_{j}^{\prime}$ and $f_{j}^{\prime}(z)$ are the $M^{\prime}$ largest eigenvalues and the corresponding eigenfunctions (KL modes) of the correlation function $C_{Y}(\Delta z)$. The coefficients $\xi_{j}^{\prime}(\theta)$ are mutually dependent non-Gaussian random variables defined 
as:

$$
\xi_{j}^{\prime}(\theta)=\frac{1}{\sqrt{\lambda_{j}^{\prime}}} \int_{D} Y(z, \theta) f_{j}^{\prime}(z) d z
$$

The exact evaluation of the integral in this equation is impossible as the complete family of joint PDFs of the random process $Y(z, \theta)$ is unknown. Therefore, a Hermite polynomial approximation of the process $Y(z, \theta)$ with a similar marginal PDF and a similar correlation function is introduced in equation (8). As opposed to the random process $Y(z, \theta)$, the approximation is completely defined from a probabilistic point of view. It is obtained according to the method presented by Sakamoto and Ghanem [7] and Puig, Poirion and Soize [8]. First, $Y(z, \theta)$ is expressed as a Hermite polynomial expansion of order $P$ in terms of an underlying standard Gaussian process $X(z, \theta)$ :

$$
Y(z, \theta) \approx \sum_{p=0}^{P} v_{p} \psi_{p}(X(z, \theta))
$$

The coefficients $v_{p}$ are found as:

$$
v_{p}=\frac{1}{\sqrt{p !}} \int_{-\infty}^{\infty} F_{Y}^{-1}\left(F_{X}(x)\right) \psi_{p}(x) p_{X}(x) d x
$$

where $F_{Y}(y)$ denotes the marginal cumulative distribution function (CDF) of $Y(z, \theta)$ and $p_{X}(x)$ and $F_{X}(x)$ denote the standard Gaussian PDF and CDF, respectively. Next, the correlation function $C_{X}(\Delta z)$ of the process $X(z, \theta)$ is determined so that the transformation (9) leads to a process with the objective correlation function $C_{Y}(\Delta z)[7,8]$. The underlying Gaussian process $X(z, \theta)$ in equation (9) is discretized by means of the $M$-th order KL decomposition:

$$
Y(z, \theta) \approx \sum_{p=0}^{P} v_{p} \psi_{p}\left(\sum_{k=1}^{M} \sqrt{\lambda_{k}} f_{k}(z) \xi_{k}(\theta)\right)
$$

Herein, $\lambda_{k}$ and $f_{k}(z)$ are the $M$ largest eigenvalues and corresponding eigenfunctions of the correlation function $C_{X}(\Delta z)$. Since $X(z, \theta)$ is a zero mean Gaussian process, the random variables $\xi_{k}(\theta)$ are mutually independent and standard Gaussian. As a result, they are easily simulated by means of a random number generator. Equation (11) is rewritten as follows in terms of the $M$-variate Hermite polynomials $\psi_{q}^{M}\left(\left\{\xi_{k}(\theta)\right\}_{k=1}^{M}\right)$ :

$$
Y(z, \theta) \approx \sum_{q=1}^{Q} w_{q}(z) \psi_{q}^{M}\left(\left\{\xi_{k}(\theta)\right\}_{k=1}^{M}\right)
$$

where $Q=\frac{(M+P) !}{M ! P !}$ denotes the number of $M$-variate Hermite polynomials of degree $P$. Equation (12) is introduced in equation (8) and the mutually 
dependent non-Gaussian coefficients $\xi_{j}^{\prime}(\theta)$ are found as:

$$
\xi_{j}^{\prime}(\theta) \approx \sum_{q=1}^{Q} d_{q j} \psi_{q}^{M}\left(\left\{\xi_{k}(\theta)\right\}_{k=1}^{M}\right)
$$

where:

$$
d_{q j}=\frac{1}{\sqrt{\lambda_{j}^{\prime}}} \int_{D} w_{q}(z) f_{j}^{\prime}(z) d z
$$

The discretization of the random process $Y(z, \theta)$ is achieved by the introduction of equation (13) in the KL decomposition (7):

$$
Y(z, \theta)=\sum_{j=1}^{M^{\prime}} \sqrt{\lambda_{j}^{\prime}} f_{j}^{\prime}(z) \sum_{q=1}^{Q} d_{q j} \psi_{q}^{M}\left(\left\{\xi_{k}(\theta)\right\}_{k=1}^{M}\right)
$$

Compared to the discretization presented in equation (12), this approach has the advantage that the number of terms in the decomposition is smaller.

\subsection{Stochastic system equations}

The application of the hybrid thin layer - direct stiffness formulation to a soil where the dynamic shear modulus is represented by a random process $G(z, \theta)=m_{G}(z)+\sigma_{G}(z) Y(z, \theta)$ in the domain $D$ leads to the following system equations:

$$
\tilde{\mathbf{K}}(\theta) \tilde{\mathbf{U}}(\theta)=\tilde{\mathbf{F}}
$$

where the stochastic stiffness matrix $\tilde{\mathbf{K}}(\theta)$ depends linearly on the random process $Y(z, \theta)$. This relation is written in operator notation as:

$$
\tilde{\mathbf{K}}(\theta)=\tilde{\mathbf{K}}_{0}+\tilde{\mathcal{K}}(Y(z, \theta))
$$

where $\tilde{\mathbf{K}}_{0}$ is the stiffness matrix of the mean system. The random process $Y(z, \theta)$ is discretized according to equation (15) and introduced in equation (17). By virtue of the linearity of $\tilde{\mathcal{K}}$, this leads to:

$$
\tilde{\mathbf{K}}(\theta)=\tilde{\mathbf{K}}_{0}+\sum_{j=1}^{M^{\prime}} \tilde{\mathbf{K}}_{j} \sum_{q=1}^{Q} d_{q j} \psi_{q}^{M}\left(\left\{\xi_{k}(\theta)\right\}_{k=1}^{M}\right)
$$

Herein, the deterministic matrices $\tilde{\mathbf{K}}_{j}$ are defined as:

$$
\tilde{\mathbf{K}}_{j}=\tilde{\mathcal{K}}\left(\sqrt{\lambda_{j}^{\prime}} f_{j}^{\prime}(x)\right)
$$

The stochastic system equations are obtained by the introduction of equation (18) in equation (16). The stochastic Green's functions $\hat{u}_{z j}^{G}(r, z, \omega, \theta)$ are 
estimated by means of a Monte Carlo simulation where $N$ realizations of $M$ independent standard Gaussian variables $\xi_{k}\left(\theta_{n}\right)$ are introduced in the stochastic system equations and the inverse Hankel transformation is applied for every realization.

\section{Results}

The stochastic Green's functions $\hat{u}_{z j}^{G}(r, z, \omega, \theta)$ due to a vertical harmonic load at the soil's surface are calculated for the site in Sint-Katelijne-Waver. The focus is on the frequency range up to $200 \mathrm{~Hz}$, which is the range of interest for rail traffic induced vibrations. The non-Gaussian process $Y(z, \theta)$ and the underlying Gaussian process $X(z, \theta)$ are discretized using a Karhunen-Loeve decomposition of order $M=M^{\prime}=15$. The relation between both processes is approximated by a Hermite polynomial expansion of order $P=2$. The stochastic system equations are formulated for a model consisting of 140 thin layers with a thickness of $h=0.10 \mathrm{~m}$ on top of a homogeneous halfspace. These equations are solved for $N=1000$ realizations. The convergence of the results with respect to these parameters is verified. In particular, the influence of the order $M$ and $M^{\prime}$ of the Karhunen-Loeve decompositions is illustrated in the following.

\subsection{Green's functions of the mean system}

In this section, the Green's functions $\hat{u}_{z j}^{G 0}(r, z, \omega)$ of the mean system due to a vertical harmonic load at the surface are discussed. The mean system consists of a vertically inhomogeneous layer with a thickness $L=14 \mathrm{~m}$, where the dynamic shear modulus $m_{G}(z)$ increases linearly, on top of a homogeneous halfspace.

Figure 4 shows the Green's functions $\hat{u}_{z j}^{G 0}(r, z, \omega)$ of the mean system at $20 \mathrm{~Hz}$ and $50 \mathrm{~Hz}$. The horizontal and the vertical components of the displacement vectors shown in the figure respectively represent the Green's functions $\hat{u}_{z r}^{G 0}(r, z, \omega)$ and $\hat{u}_{z z}^{G 0}(r, z, \omega)$ on a grid of receivers in the $(r, z)$-plane. Along the axis of symmetry $r=0 \mathrm{~m}$, the motion consists of a dilatational wave. The amplitude of this wave is small as the Poisson's ratio $\nu=0.47$ is close to 0.5 and the soil has a low compressibility. A shear window is travelling in an average direction of $45^{\circ}$. The shear wavelength $\lambda_{s}$ depends on the depth $z$ due to the spatial variation of the dynamic shear modulus $m_{G}(z)$. For a depth $z=10 \mathrm{~m}$ and a frequency $f=50 \mathrm{~Hz}$, the shear wavelength is $\lambda_{s}=9.1 \mathrm{~m}$, as confirmed by figure $4 \mathrm{~b}$. In the region near the soil's surface, the response $\hat{u}_{z j}^{G 0}(r, z, \omega)$ is dominated by the Rayleigh wave and the particle motion is el- 


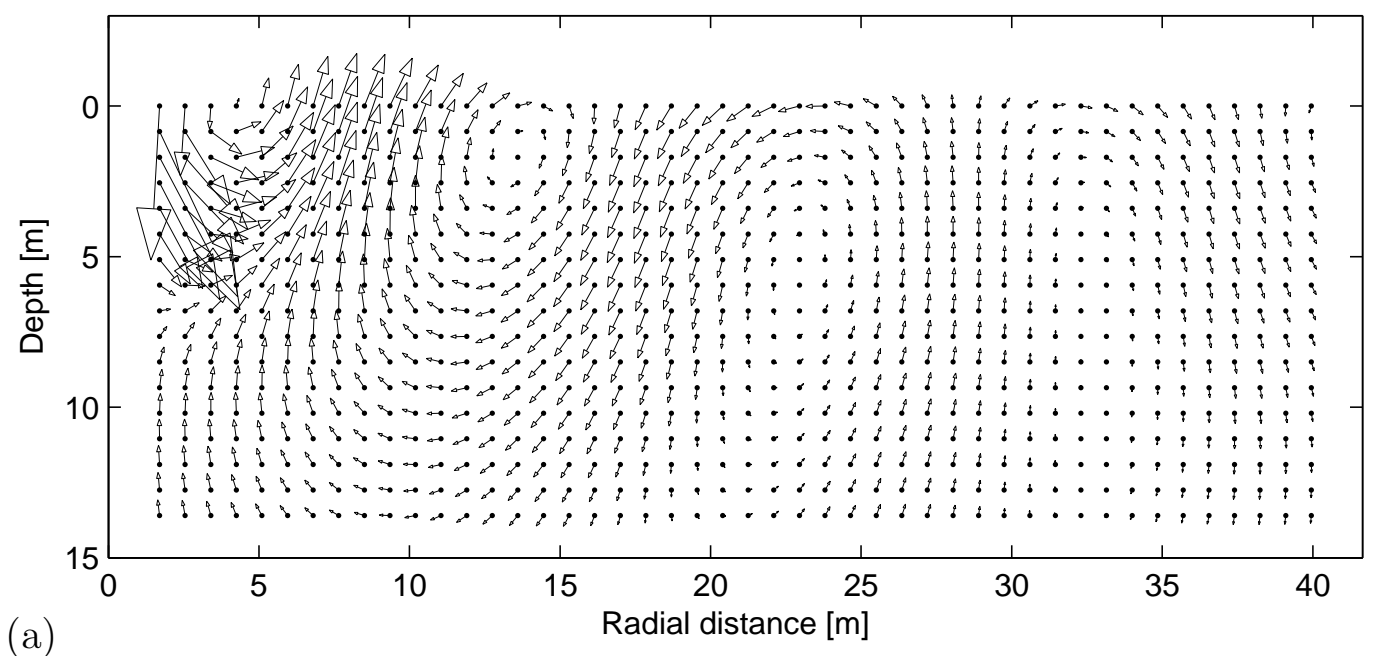

(a)

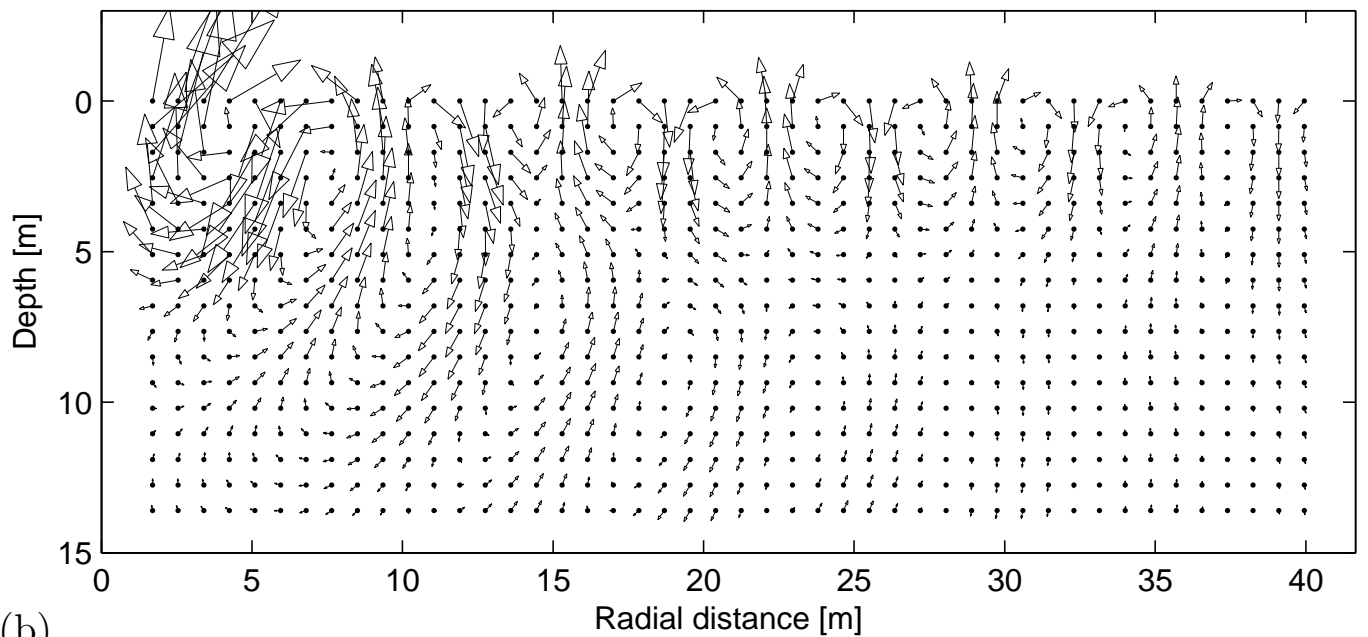

(b)

Radial distance [m]

Fig. 4. Real part of the Green's functions $\hat{u}_{z j}^{G 0}(r, z, \omega)$ of the mean system at (a) $20 \mathrm{~Hz}$ and (b) $50 \mathrm{~Hz}$.

liptical and counterclockwise. The motion decays exponentially with depth and almost disappears at a depth of two times the Rayleigh wavelength. The largest displacements are observed close to the surface up to a depth $z=\gamma \lambda_{R}$ where $\gamma \approx 0.3$. The Rayleigh wavelength $\lambda_{R}$ is close to the shear wavelength $\lambda_{s}$ at the surface: $\lambda_{R} \approx 15.1 \mathrm{~m}$ for $f=20 \mathrm{~Hz}$ and $\lambda_{R} \approx 6.0 \mathrm{~m}$ for $f=50 \mathrm{~Hz}$.

Figure 5 shows the frequency content of the Green's function $\hat{u}_{z z}^{G 0}(r, z, \omega)$ of the mean system at six different positions. The response $\hat{u}_{z z}^{G 0}(r, z, \omega)$ decreases as the source-receiver distance $r$ increases due to both geometrical and material damping. The attenuation due to geometrical damping is frequency independent, while the attenuation due to material damping is larger in the high frequency range. For a fixed radial distance $r$, the largest response $\hat{u}_{z z}^{G 0}(r, z, \omega)$ is observed at the soil's surface where the Rayleigh wave dominates and the dynamic shear modulus $m_{G}(z)$ reaches a minimum value. The oscillation of the response $\hat{u}_{z z}^{G 0}(r, z, \omega)$ as a function of the frequency is due to the interference of reflected and refracted waves induced by the spatial variation of the 


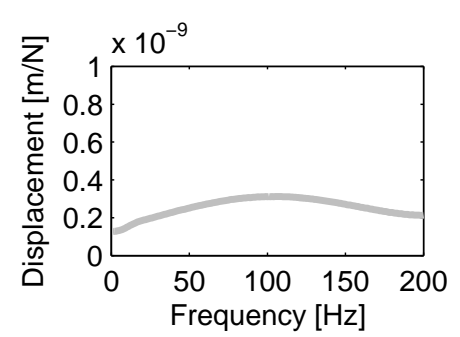

(a) $r=2 \mathrm{~m}, z=0 \mathrm{~m}$

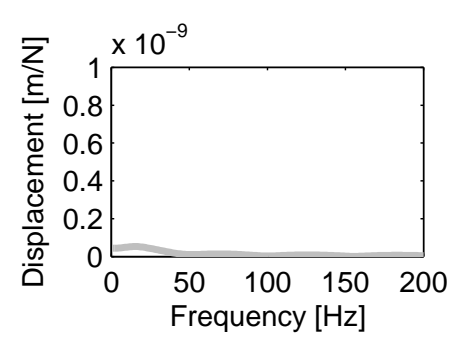

(d) $r=2 \mathrm{~m}, z=8 \mathrm{~m}$

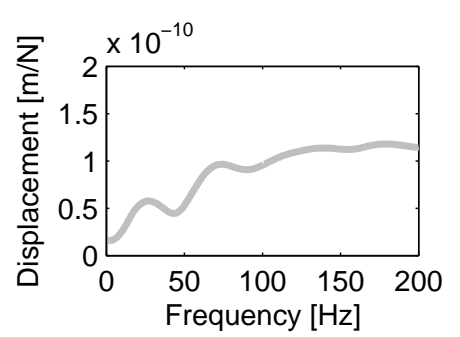

(b) $r=10 \mathrm{~m}, z=0 \mathrm{~m}$

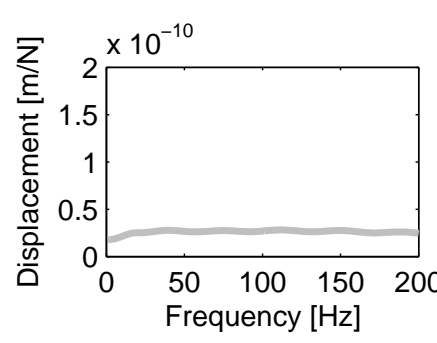

(e) $r=10 \mathrm{~m}, z=8 \mathrm{~m}$

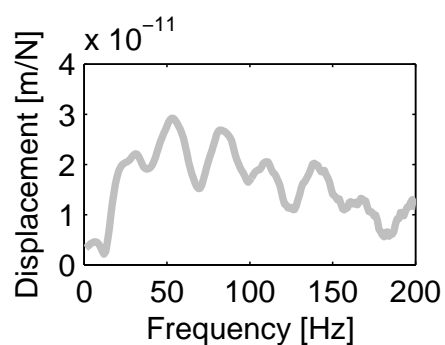

(c) $r=50 \mathrm{~m}, z=0 \mathrm{~m}$

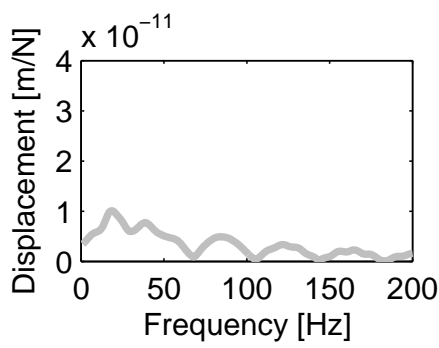

(f) $r=50 \mathrm{~m}, z=8 \mathrm{~m}$

Fig. 5. Modulus of the Green's function $\hat{u}_{z z}^{G 0}(r, z, \omega)$ of the mean system at the points (a) $r=2 \mathrm{~m}, z=0 \mathrm{~m}$, (b) $r=10 \mathrm{~m}, z=0 \mathrm{~m}$, (c) $r=50 \mathrm{~m}, z=0 \mathrm{~m}$, (d) $r=2 \mathrm{~m}, z=8 \mathrm{~m}$, (e) $r=10 \mathrm{~m}, z=8 \mathrm{~m}$, and (f) $r=50 \mathrm{~m}, z=8 \mathrm{~m}$.

dynamic shear modulus $m_{G}(z)$.

\subsection{Realizations of the stochastic Green's functions}

Figure 6 shows five realizations of the stochastic Green's functions $\hat{u}_{z j}^{G}\left(r, z, \omega, \theta_{i}\right)$ corresponding to five randomly chosen realizations of the stochastic dynamic shear modulus $G\left(z, \theta_{i}\right)$. The index $i$ refers to the $i$-th realization.

At depth, the motion is dominated by the vertical dilatational wave and the shear waves. The dynamic shear modulus $G(z, \theta)$ varies randomly along the propagation path of both waves. In the frequency range up to $50 \mathrm{~Hz}$, the longitudinal wavelength $\lambda_{p}$ and the shear wavelength $\lambda_{s}$ are large compared to the correlation length $l_{c}$ of the dynamic shear modulus $G(z, \theta)$. Consequently, the waves do not resolve the small scale variations of the dynamic shear modulus and the soil behaves as the mean system. This is confirmed by figure 6: at depth, the realizations $\hat{u}_{z j}^{G}\left(r, z, \omega, \theta_{i}\right)$ are similar to each other and to the Green's functions $\hat{u}_{z j}^{G 0}(r, z, \omega)$ of the mean system shown in figure 4 . The strongest similarity is observed at $20 \mathrm{~Hz}$ where the wavelengths $\lambda_{p}$ and $\lambda_{s}$ are large.

Near the surface, the motion is dominated by the Rayleigh wave. The dynamic shear modulus $G(z, \theta)$ does not vary along the propagation path of this wave. 

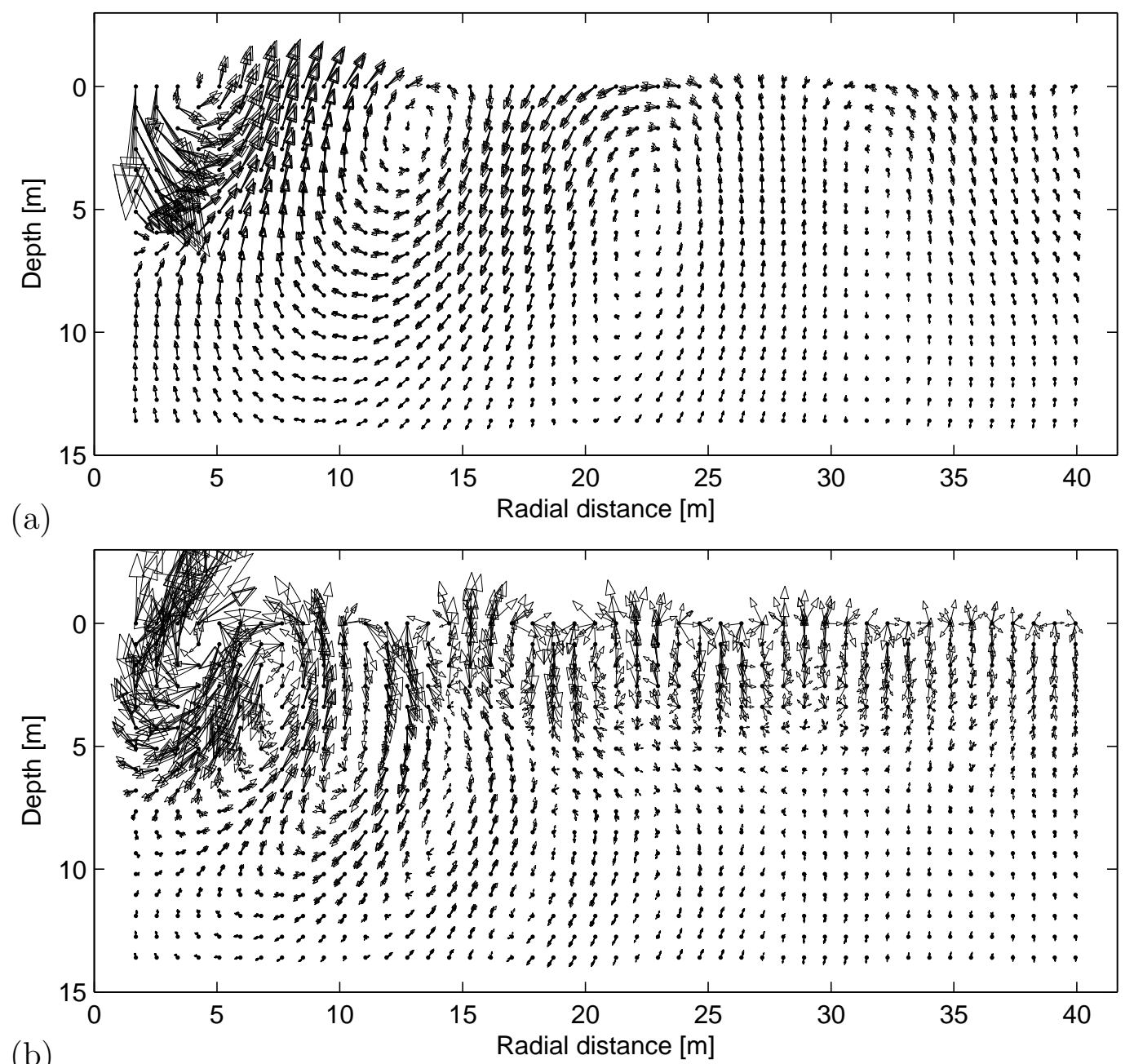

Radial distance $[\mathrm{m}]$

Fig. 6. Real part of five realizations of the stochastic Green's functions $\hat{u}_{z j}^{G}\left(r, z, \omega, \theta_{i}\right)$ at (a) $20 \mathrm{~Hz}$ and (b) $50 \mathrm{~Hz}$.

The influence of the heterogeneity of the soil is still related to the ratio of the wavelength $\lambda_{R}$ and the correlation length $l_{c}$, however. The Rayleigh wave involves large displacements in a region near the surface with a depth $z=\gamma \lambda_{R}$. The average soil characteristics in this region control the contribution of the Rayleigh wave to the displacement at the soil's surface. For low frequencies, the depth $z=\gamma \lambda_{R}$ is large compared to the correlation length $l_{c}$ of the dynamic shear modulus $G(z, \theta)$. The spatial average of the dynamic shear modulus $G(z, \theta)$ up to a depth $z=\gamma \lambda_{R}$ is similar for every realization, independent of the small scale variations of the dynamic shear modulus. As a result, the realizations of the Green's functions $\hat{u}_{z j}^{G}\left(r, z, \omega, \theta_{i}\right)$ shown in figure $6 \mathrm{a}$ and the Green's functions $\hat{u}_{z j}^{G 0}(r, z, \omega)$ of the mean system shown in figure 4a are similar to each other both at depth and near the surface. For high frequencies, the depth $z=\gamma \lambda_{R}$ is small compared to the correlation length $l_{c}$ and the spatial average of the dynamic shear modulus $G(z, \theta)$ up to a depth $z=\gamma \lambda_{R}$ differs for every realization. As a consequence, the realizations of the Green's functions 
$\hat{u}_{z j}^{G}\left(r, z, \omega, \theta_{i}\right)$ shown in figure $6 \mathrm{~b}$ clearly differ from each other and from the Green's functions $\hat{u}_{z j}^{G 0}(r, z, \omega)$ of the mean system shown in figure $4 \mathrm{~b}$. The difference emerges in both the amplitude and the phase of the Green's function in the region near the surface. The amplitude is affected by the variability of the dynamic shear modulus $G(z, \theta)$ which reflects the stiffness of the soil. The phase is affected via the wave velocities that differ for every realization. Due to the homogeneity of the soil in the horizontal direction, an accumulation of the phase shifts between different realizations occurs as the ratio of the horizontal source-receiver distance and the wavelength increases. This loss of coherence is confirmed by figure $6 \mathrm{~b}$ where all realizations are almost in-phase at positions close to the source, while phase shifts up to $180^{\circ}$ occur at a radial distance $r=40 \mathrm{~m}$.

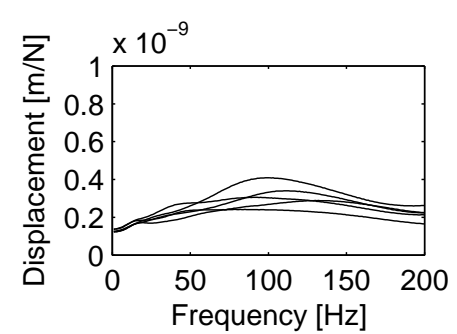

(a) $r=2 \mathrm{~m}, z=0 \mathrm{~m}$

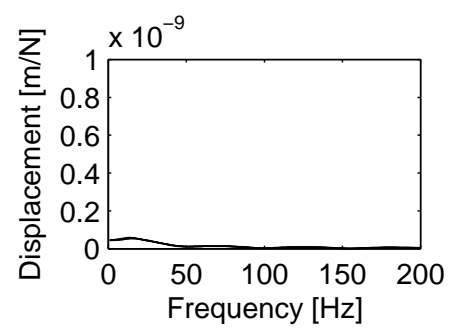

(d) $r=2 \mathrm{~m}, z=8 \mathrm{~m}$

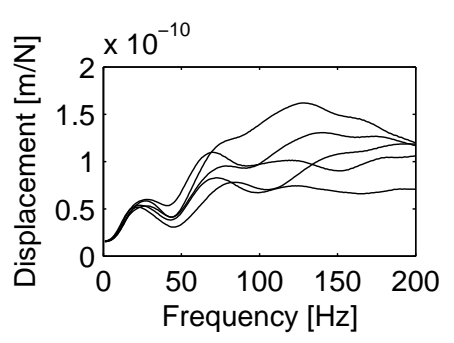

(b) $r=10 \mathrm{~m}, z=0 \mathrm{~m}$

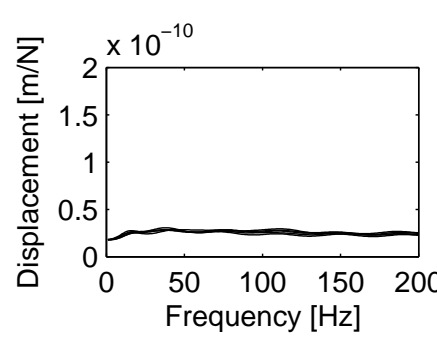

(e) $r=10 \mathrm{~m}, z=8 \mathrm{~m}$

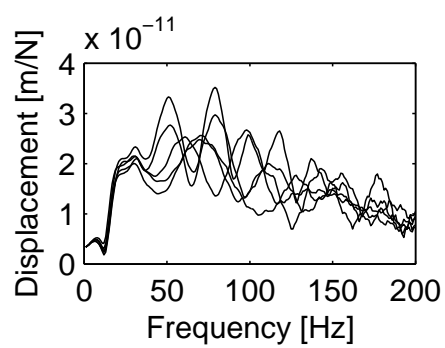

(c) $r=50 \mathrm{~m}, z=0 \mathrm{~m}$

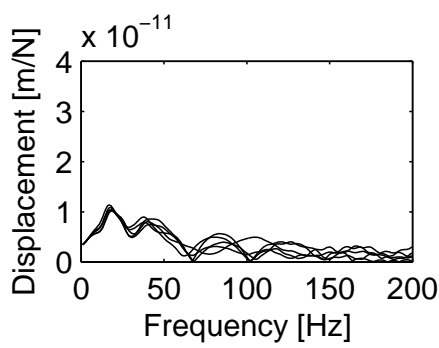

(f) $r=50 \mathrm{~m}, z=8 \mathrm{~m}$

Fig. 7. Modulus of five realizations of the stochastic Green's function $\hat{u}_{z z}^{G}\left(r, z, \omega, \theta_{i}\right)$ at the points (a) $r=2 \mathrm{~m}, z=0 \mathrm{~m}$, (b) $r=10 \mathrm{~m}, z=0 \mathrm{~m}$, (c) $r=50 \mathrm{~m}, z=0 \mathrm{~m}$, (d) $r=2 \mathrm{~m}, z=8 \mathrm{~m}$, (e) $r=10 \mathrm{~m}, z=8 \mathrm{~m}$, and (f) $r=50 \mathrm{~m}, z=8 \mathrm{~m}$.

Figure 7 shows the frequency content of the realizations of the stochastic Green's function $\hat{u}_{z z}^{G}\left(r, z, \omega, \theta_{i}\right)$ at six different positions. At depth, where the vertical dilatational wave and the shear waves dominate the response, the variability is small in the frequency range up to $200 \mathrm{~Hz}$. At the surface, where the Rayleigh wave propagates, the variability of the response increases as the frequency increases. The oscillatory behavior due to the occurrence of reflected and refracted waves is observed for every realization. The oscillation pattern shifts along the frequency axis due to the variability of the wave velocities.

Figures 8 and 9 illustrate the influence of the order of the KL decomposition used to discretize the dynamic shear modulus $G(z, \theta)$. Figure 8 shows five realizations of the stochastic dynamic shear modulus $G\left(z, \theta_{i}\right)$, obtained with 


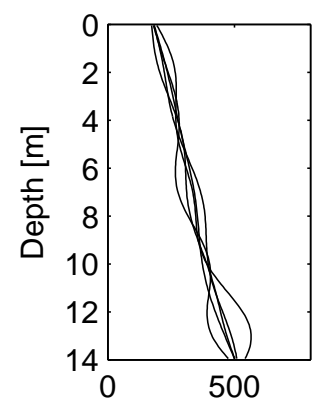

(a)

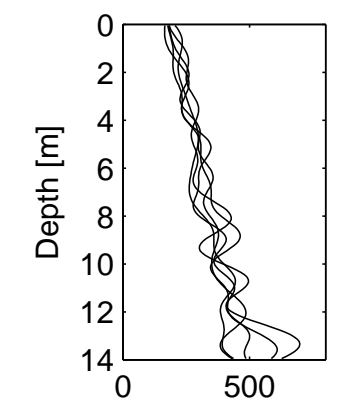

(b)

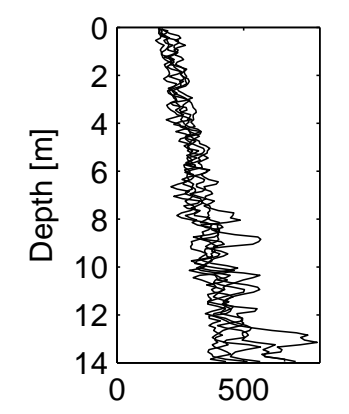

(c)

Fig. 8. Five realizations of the stochastic dynamic shear modulus $G\left(z, \theta_{i}\right)$, obtained with a Karhunen-Loeve decomposition of order (a) $M=M^{\prime}=5$, (b) $M=M^{\prime}=15$, and (c) $M=M^{\prime}=100$.

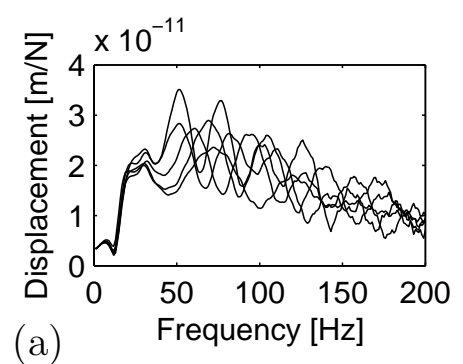

(a)

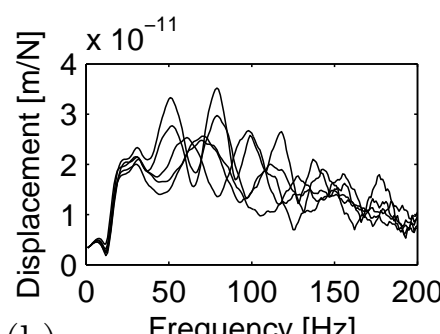

(b)

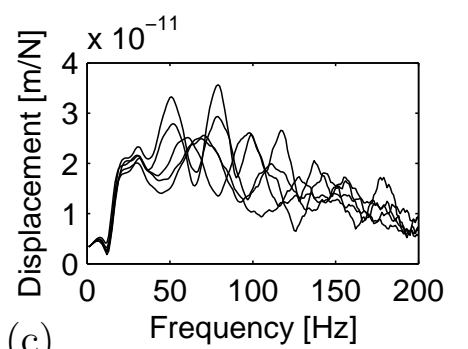

(c)

Fig. 9. Modulus of five realizations of the stochastic Green's function $\hat{u}_{z z}^{G}\left(r, z, \omega, \theta_{i}\right)$ at the point $r=50 \mathrm{~m}, z=0 \mathrm{~m}$, obtained with a Karhunen-Loeve decomposition of order (a) $M=M^{\prime}=5$, (b) $M=M^{\prime}=15$, and (c) $M=M^{\prime}=100$.

a KL decomposition of order $M=M^{\prime}=5,15$, and 100. Figure 9 shows the modulus of the corresponding realizations of the stochastic Green's function $\hat{u}_{z z}^{G}\left(r, z, \omega, \theta_{i}\right)$. Comparison of figures $8 \mathrm{a}, 8 \mathrm{~b}$, and $8 \mathrm{c}$ reveals that the variance of the random process $G(z, \theta)$ is not fully accounted for in the case where $M=$ $M^{\prime}=5$ or 15 , due to the absence of the highest KL modes that represent the smallest scale variations. As a result, the realizations of the Green's function $\hat{u}_{z z}^{G}\left(r, z, \omega, \theta_{i}\right)$ obtained with a KL decomposition of order $M=M^{\prime}=5$ (figure 9a) differ from the realizations obtained with $M=M^{\prime}=15$ (figure 9b). While the difference is obvious in the high frequency range, it almost disappears in the low frequency range where the waves only resolve the first $5 \mathrm{KL}$ modes. Similarly, only the first $15 \mathrm{KL}$ modes are resolved in the frequency range up to $200 \mathrm{~Hz}$ and the difference between the Green's function obtained with a KL decomposition of order $M=M^{\prime}=15$ (figure $9 \mathrm{~b}$ ) and with $M=M^{\prime}=$ 100 (figure 9c) is negligible. The requirements for $M$ and $M^{\prime}$ to obtain an appropriate estimation of the stochastic Green's functions up to $200 \mathrm{~Hz}$ are consequently less strict than for the simulation of the process $G(z, \theta)$ itself. 


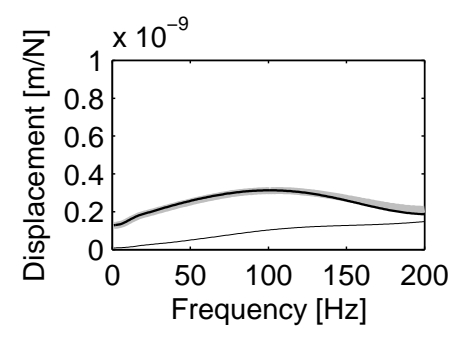

(a) $r=2 \mathrm{~m}, z=0 \mathrm{~m}$

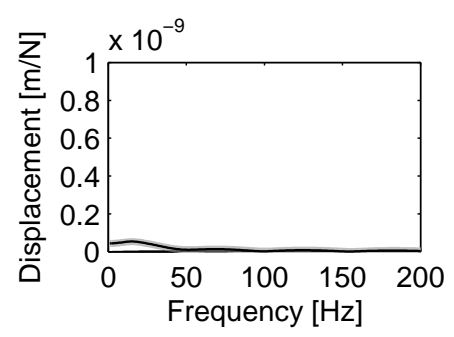

(d) $r=2 \mathrm{~m}, z=8 \mathrm{~m}$

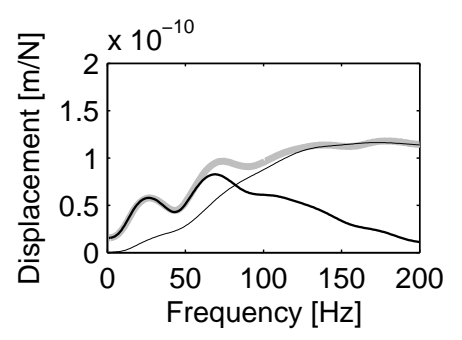

(b) $r=10 \mathrm{~m}, z=0 \mathrm{~m}$

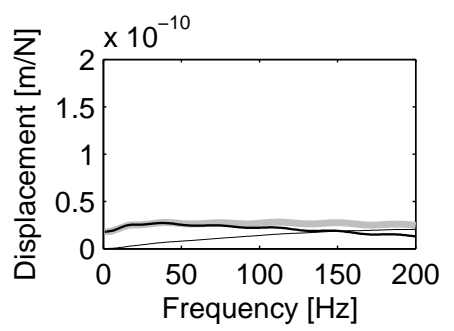

(e) $r=10 \mathrm{~m}, z=8 \mathrm{~m}$

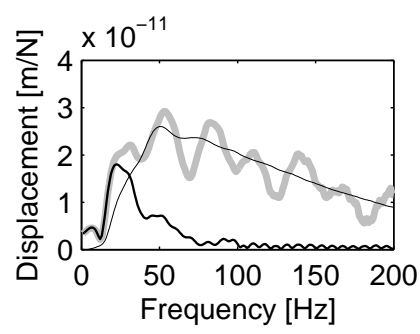

(c) $r=50 \mathrm{~m}, z=0 \mathrm{~m}$

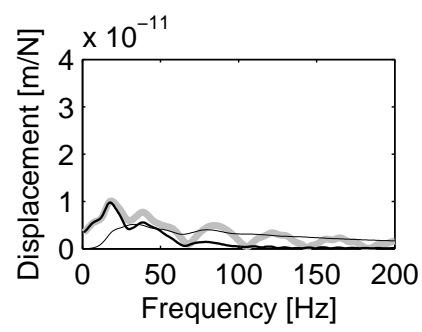

(f) $r=50 \mathrm{~m}, z=8 \mathrm{~m}$

Fig. 10. Modulus of the Green's function $\hat{u}_{z z}^{G 0}(r, z, \omega)$ of the mean system $(-)$, modulus of the mean value $m_{\hat{u}_{z z}^{G}}(r, z, \omega)$ of the stochastic Green's function (-) and standard deviation $\sigma_{\hat{u}_{z z}^{G}}(r, z, \omega)$ of the stochastic Green's function (-) at the points (a) $r=2 \mathrm{~m}, z=0 \mathrm{~m}$, (b) $r=10 \mathrm{~m}, z=0 \mathrm{~m}$, (c) $r=50 \mathrm{~m}, z=0 \mathrm{~m}$, (d) $r=2 \mathrm{~m}, z=8 \mathrm{~m}$, (e) $r=10 \mathrm{~m}, z=8 \mathrm{~m}$, and (f) $r=50 \mathrm{~m}, z=8 \mathrm{~m}$.

\subsection{Statistics of the stochastic Green's functions}

Figure 10 shows the modulus of the mean value $m_{\hat{u}_{z z}^{G}}(r, z, \omega)$ and the standard deviation $\sigma_{\hat{u}_{z z}^{G}}(r, z, \omega)$ of the stochastic Green's function $\hat{u}_{z z}^{G}(r, z, \omega, \theta)$, as well as the Green's function $\hat{u}_{z z}^{G 0}(r, z, \omega)$ of the mean system, at six different positions.

In the low frequency range, the wavelengths are large and the small scale variations of the dynamic shear modulus do not influence the waves. As a result, the random medium is satisfactorily described by the mean soil model: the Green's function $\hat{u}_{z z}^{G 0}(r, z, \omega)$ of the mean system is close to the mean Green's function $m_{\hat{u}_{z z}^{G}}(r, z, \omega)$ and the standard deviation $\sigma_{\hat{u}_{z z}^{G}}(r, z, \omega)$ of the stochastic Green's function is small.

In the high frequency range, the mean Green's function $m_{\hat{u}_{z z}^{G}}(r, z, \omega)$ is clearly attenuated. This is due the loss of coherence between different realizations of the Green's function $\hat{u}_{z z}^{G}\left(r, z, \omega, \theta_{i}\right)$ : out-of-phase realizations cancel out each other. The effect intensifies as the ratio of the source-receiver distance and the wavelength increases, due to the corresponding accumulation of phase shifts between the realizations. The attenuation of the mean Green's function $m_{\hat{u}_{z z}^{G}}(r, z, \omega)$ is balanced by an increase of the Green's function's standard deviation $\sigma_{\hat{u}_{z z}^{G}}(r, z, \omega)$, indicating a shift of energy from the mean value to 
the standard deviation rather than the absorption of energy. As a result, the mean Green's function $m_{\hat{u}_{z z}^{G}}(r, z, \omega)$ is no longer representative for the response observed for a single realization and the statistics $m_{\hat{u}_{z z}^{G}}(r, z, \omega)$ and $\sigma_{\hat{u}_{z z}^{G}}(r, z, \omega)$ do not allow one to assess the vibration levels in the free field.
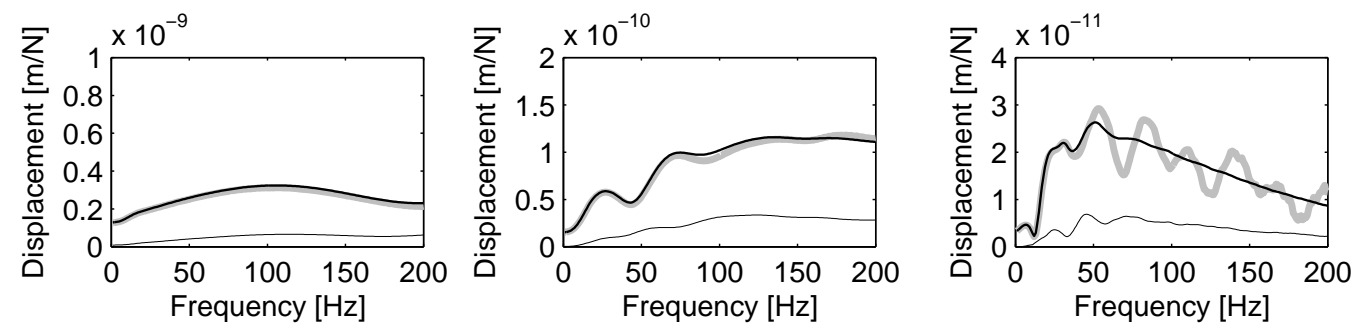

(a) $r=2 \mathrm{~m}, z=0 \mathrm{~m}$

(b) $r=10 \mathrm{~m}, z=0 \mathrm{~m}$

(c) $r=50 \mathrm{~m}, z=0 \mathrm{~m}$
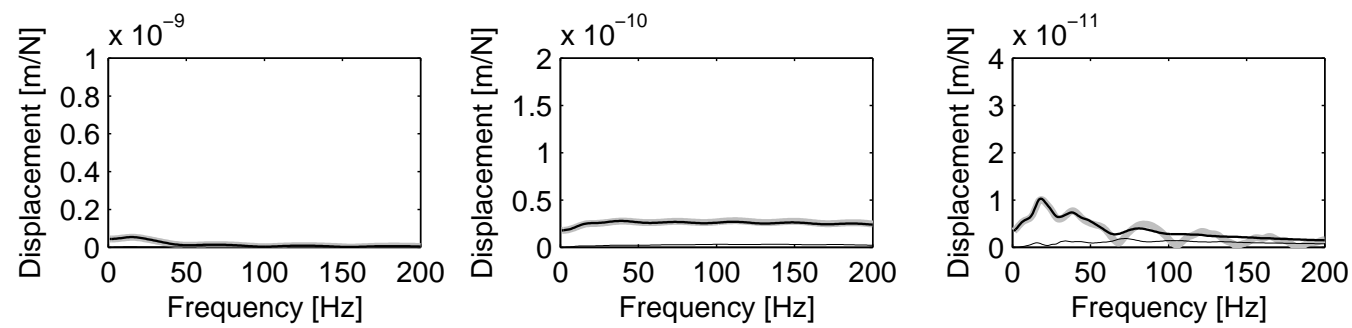

(d) $r=2 \mathrm{~m}, z=8 \mathrm{~m}$

(e) $r=10 \mathrm{~m}, z=8 \mathrm{~m}$

(f) $r=50 \mathrm{~m}, z=8 \mathrm{~m}$

Fig. 11. Modulus of the Green's function $\hat{u}_{z z}^{G 0}(r, z, \omega)$ of the mean system $(-)$, mean value $m_{\left|\hat{u}_{z z}^{G}\right|}(r, z, \omega)$ of the modulus of the stochastic Green's function (-) and standard deviation $\sigma_{\left|\hat{u}_{z z}^{G}\right|}(r, z, \omega)$ of the modulus of the stochastic Green's function (-) at the points (a) $r=2 \mathrm{~m}, z=0 \mathrm{~m}$, (b) $r=10 \mathrm{~m}, z=0 \mathrm{~m}$, (c) $r=50 \mathrm{~m}, z=0 \mathrm{~m}$, (d) $r=2 \mathrm{~m}, z=8 \mathrm{~m}$, (e) $r=10 \mathrm{~m}, z=8 \mathrm{~m}$, and (f) $r=50 \mathrm{~m}, z=8 \mathrm{~m}$.

The assessment of the free field vibration levels is possible using the statistics $m_{\left|\hat{u}_{z z}^{G}\right|}(r, z, \omega)$ and $\sigma_{\left|\hat{u}_{z z}^{G}\right|}(r, z, \omega)$ of the modulus of the stochastic Green's function $\hat{u}_{z z}^{G}(r, z, \omega, \theta)$. These statistics are shown in figure 11. In the entire frequency range, a good correspondence of the mean modulus of the stochastic Green's function and the modulus of the Green's function of the mean system is observed, although the oscillatory pattern in the latter does not occur in the former. At low frequencies, the small scale variations of the dynamic shear modulus do not influence the waves and the standard deviation $\sigma_{\left|\hat{u}_{z z}^{G}\right|}(r, z, \omega)$ of the modulus of the Green's function is small. As the frequency increases, the effect of the small scale variations develops and the standard deviation $\sigma_{\left|\hat{u} \hat{G}_{z}\right|}(r, z, \omega)$ increases. For a fixed frequency, the variability of the Green's function modulus, reflected by the coefficient of variation $\sigma_{\left|\hat{u}_{z z}^{G}\right|}(r, z, \omega) / m_{\left|\hat{u}_{z z}^{G}\right|}(r, z, \omega)$, is larger at the surface than at depth.

A more appealing way to present the variability of the modulus of the Green's function is provided by the confidence region. The confidence region with a probability $P_{c}=0.95$ is estimated via the marginal CDF of the Green's function using the results of the Monte Carlo simulation and shown in figure 12. Analogously to the standard deviation $\sigma_{\left|\hat{u}_{z z}^{G}\right|}(r, z, \omega)$ shown in figure 11 , 


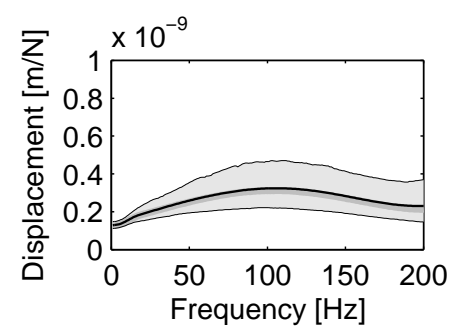

(a) $r=2 \mathrm{~m}, z=0 \mathrm{~m}$

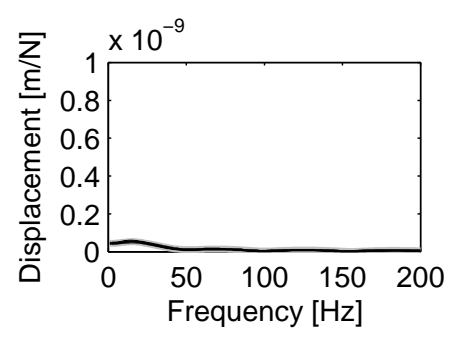

(d) $r=2 \mathrm{~m}, z=8 \mathrm{~m}$

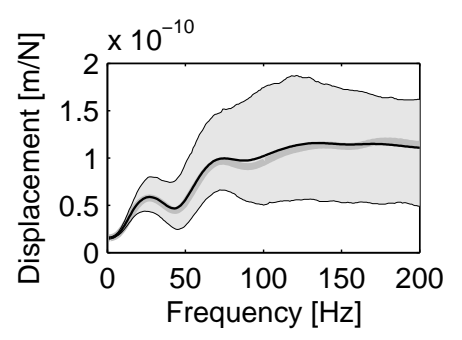

(b) $r=10 \mathrm{~m}, z=0 \mathrm{~m}$

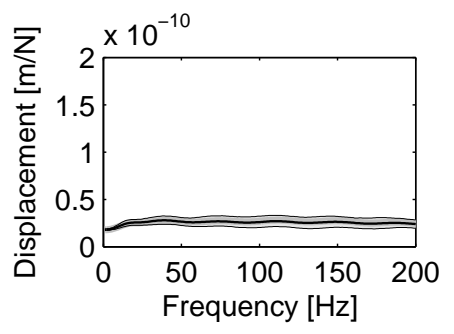

(e) $r=10 \mathrm{~m}, z=8 \mathrm{~m}$

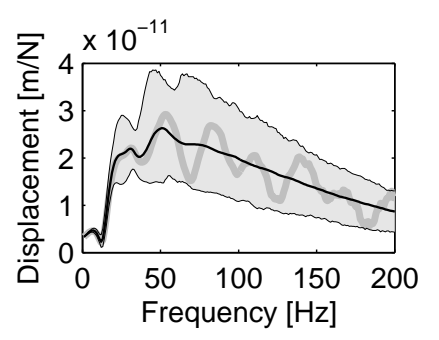

(c) $r=50 \mathrm{~m}, z=0 \mathrm{~m}$

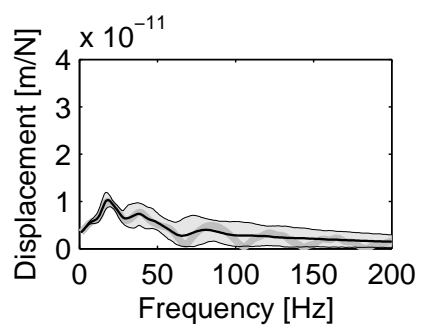

(f) $r=50 \mathrm{~m}, z=8 \mathrm{~m}$

Fig. 12. Modulus of the Green's function $\hat{u}_{z z}^{G 0}(r, z, \omega)$ of the mean system $(-)$, mean value $m_{\left|\hat{u}_{z z}^{G}\right|}(r, z, \omega)$ of the modulus of the stochastic Green's function (-) and confidence bounds of the modulus of the stochastic Green's function (-) at the points (a) $r=2 \mathrm{~m}, z=0 \mathrm{~m}$, (b) $r=10 \mathrm{~m}, z=0 \mathrm{~m}$, (c) $r=50 \mathrm{~m}, z=0 \mathrm{~m}$, (d) $r=2 \mathrm{~m}, z=8 \mathrm{~m}$, (e) $r=10 \mathrm{~m}, z=8 \mathrm{~m}$, and (f) $r=50 \mathrm{~m}, z=8 \mathrm{~m}$. The probability of the confidence region is $P_{c}=0.95$.

the width of the confidence region is small in the low frequency range and increases with the frequency. For a fixed frequency, the ratio of the width of the confidence region and the mean Green's function modulus $m_{\left|\hat{u}_{z z}^{G}\right|}(r, z, \omega)$ is larger at the surface than at depth.

Figures 11 and 12 allow one to conclude that the mean system provides an accurate prediction of the mean modulus of the stochastic Green's functions in the frequency range up to $200 \mathrm{~Hz}$. In the low frequency range, the additional random component of the modulus of the stochastic Green's functions is small so that the mean system allows for reliable predictions of the vibration levels in the free field. In the high frequency range, the importance of this random component increases and a stochastic soil model is required. This conclusion concurs with the discrepancy observed between the measured and the predicted vibration levels obtained with a deterministic prediction model for rail traffic induced vibrations. This does not prove that the discrepancy is due to the type of random perturbations of the soil properties considered in the present paper, however. Other types of perturbations (e.g. rocks, voids, or undulation in the layers) could lead to similar results as those obtained for a random variation of the shear modulus with depth. 


\section{Conclusion}

The Green's functions are calculated for a vertically inhomogeneous soil where the dynamic shear modulus is represented by a random process characterized by a marginal probability density function and a correlation function. The stochastic finite element method is applied to a hybrid thin layer - direct stiffness formulation in order to obtain the stochastic system equations. These equations are solved by means of a Monte Carlo simulation. A loss of coherence of the Green's functions of the individual realizations occurs if the ratio of the source-receiver distance and the wavelength is large, resulting in an attenuation of the mean Green's functions. Due to this attenuation, the mean Green's functions are not representative for the response observed for a single realization, as opposed to the mean modulus of the Green's functions which is not affected by the loss of coherence. In the low frequency range, the waves do not resolve the small scale variations of the dynamic shear modulus and the random medium is satisfactorily described by the mean soil model. In the high frequency range, the mean soil model still provides an accurate approximation of the mean modulus of the Green's functions. The additional random component becomes important, however, and a stochastic soil model is needed for a reliable prediction of the vibration levels in the free field.

\section{References}

[1] G. Lombaert, G. Degrande, D. Clouteau, Numerical modelling of free field traffic induced vibrations, Soil Dynamics and Earthquake Engineering 19 (7) (2000) 473-488.

[2] L. Pyl, G. Degrande, G. Lombaert, W. Haegeman, Validation of a sourcereceiver model for road traffic induced vibrations in buildings. I: Source model, Journal of Engineering Mechanics, Proceedings of the ASCE 130 (12) (2004) $1377-1393$.

[3] L. Pyl, G. Degrande, D. Clouteau, Validation of a source-receiver model for road traffic induced vibrations in buildings. II: Receiver model, Journal of Engineering Mechanics, Proceedings of the ASCE 130 (12) (2004) 1394-1406.

[4] G. Lombaert, G. Degrande, The experimental validation of a numerical model for the prediction of the vibrations in the free field produced by road traffic, Journal of Sound and Vibration 262 (2003) 309-331.

[5] G. Fenton, Random field modeling of CPT data, Journal of Geotechnical and Geoenvironmental Engineering, Proceedings of the ASCE 125 (6) (1999) 486535.

[6] R. Ghanem, P. Spanos, Stochastic finite elements: a spectral approach, SpringerVerlag, New York, 1991. 
[7] S. Sakamoto, R. Ghanem, Simulation of multi-dimensional non-Gaussian nonstationary random fields, Probabilistic Engineering Mechanics 17 (2002) 167176.

[8] B. Puig, F. Poirion, C. Soize, Non-Gaussian simulation using Hermite polynomial expansion: convergences and algorithms, Probabilistic Engineering Mechanics 17 (2002) 253-264.

[9] C. Yeh, M. Rahman, Stochastic finite element methods for the seismic response of soils, International Journal for Numerical and Analytical Methods in Geomechanics 22 (10) (1998) 819-850.

[10] D. Ghiocel, R. Ghanem, Stochastic finite-element analysis of seismic soilstructure interaction, Journal of Engineering Mechanics, Proceedings of the ASCE 128 (1) (2002) 66-77.

[11] S. Liao, J. Li, A stochastic approach to site-response component in seismic ground motion coherency model, Soil Dynamics and Earthquake Engineering $22(9-12)(2002)$ 813-820.

[12] A. Nour, A. Slimani, N. Laouami, H. Afra, Finite element model for the probabilistic seismic response of heterogeneous soil profile, Soil Dynamics and Earthquake Engineering 23 (5) (2003) 331-348.

[13] E. Kausel, J. Roësset, Stiffness matrices for layered soils, Bulletin of the Seismological Society of America 71 (6) (1981) 1743-1761.

[14] P. Mengé, Soil investigation results at Sint-Katelijne-Waver (Belgium), in: Proceedings of the Symposium on Screw Piles, Brussels, 2001, pp. 19-62.

[15] G. Fenton, Estimation for stochastic soil models, Journal of Geotechnical and Geoenvironmental Engineering, Proceedings of the ASCE 125 (6) (1999) 470485 .

[16] P. Mayne, G. Rix, Correlations between shear wave velocity and cone tip resistance in natural clays, Soils and Foundations 35 (2) (1995) 107-110.

[17] E. Kausel, Fundamental solutions in elastodynamics: a compendium, Cambridge University Press, New York, 2006.

[18] M. Van Veldhuizen, R. Nieuwenhuizen, W. Zijl, A note on log scale Hankel transforms, Journal of Computational Physics 110 (1) (1994) 196-199.

[19] J. Talman, Numerical Fourier and Bessel transforms in logarithmic variables, Journal of Computational Physics 29 (1) (1978) 35-48. 